See discussions, stats, and author profiles for this publication at: https://www.researchgate.net/publication/336103905

\title{
Blockchain applicability using Smart Power Management: SealedGrid Architecture
}

Conference Paper · October 2019

CITATIONS

0

2 authors:

(6) George Suciu

Polytechnic University of Bucharest

183 PUBLICATIONS 659 CITATIONS

SEE PROFILE
READS

72

Marius Vochin

Polytechnic University of Bucharest

53 PUBLICATIONS 82 CITATIONS

SEE PROFILE

Some of the authors of this publication are also working on these related projects:

EU H2020 SWITCH (Software Workbench for Interactive, Time Critical and Highly self-adaptive Cloud applications) View project

DISEDAN (Service and user-based distributed selection of content streaming source and dual adaptation) View project 


\section{Blockchain applicability using Smart Power Management: SealedGrid Architecture}

\author{
George Suciu \\ $R \& D$ Department \\ BEIA Consult International \\ Bucharest, Romania \\ george.beia.ro \\ Marius Dobrea \\ $R \& D$ Department \\ BEIA Consult International \\ Bucharest, Romania \\ marius.dobrea@beia.ro
}

\author{
Mari-Anais Sachian \\ $R \& D$ Department \\ BEIA Consult International \\ Bucharest, Romania \\ anais.sachian@beia.ro \\ Cristian Beceanu \\ $R \& D$ Department \\ BEIA Consult International \\ Bucharest, Romania \\ cristian.beceanu@beia.ro \\ Ana Petrache \\ $R \& D$ Department \\ BEIA Consult International \\ Bucharest, Romania \\ ana.petrache@beia.ro
}

\author{
Marius Vochin \\ Telecommunications Department \\ University POLITEHNICA of Bucharest \\ Bucharest, Romania \\ marius.vochin@upb.ro \\ Raluca Iosu \\ $R \& D$ Department \\ BEIA Consult International \\ Bucharest, Romania \\ raluca.iosu@beia.ro
}

\begin{abstract}
With the discovery and novelty regarding crypto mining farms, it has been foreseen that bitcoin mining and other types of cryptocurrency businesses will flourish in the near future. However, one main issue in smart grid systems is the security regarding data bases that must be addressed. Blockchain has been proven to be a safe alternative to be used in mining systems because it allows a secure applicability in databases and also another important aspect is that each hash in a crypto mining system cannot be changed if it has such an algorithm behind its build. Thus, resulting in a secure and reliable system. The purpose of this paper aims to show how blockchain can affect and be used in a smart power management system going forth from the SealedGrid platform. This system enables the user to monitor in real time the power usage in a smart grid system, in so much that this platform is built to be secure and safe from third-party attacks. Hence this paper shows how a crypto mining application can be applied to an energy saving system.
\end{abstract}

Index Terms-- Blockchain, Security, Ethereum, Smart Power Management

\section{INTRODUCTION}

This paper presents the applicability of Blockchain technology in the case of a photovoltaic panel system that supplies a building and the surplus energy that remains to be used for data mining. The photovoltaic systems (PV) used in other countries are discussed to be implemented with the integration of a decentralized distribution network using Blockchain. Energy systems are undergoing rapid transformations in increasing the number of renewable energy sources such as PV and solar energy. In 2016 it was observed that $24.6 \%$ of gross electricity consumption in the United Kingdom was generated by Renewable Energy Sources (RES), mainly from wind farms onshore and offshore, and photovoltaic panels, representing $44.9 \%$ and $12.5 \%$ of the total 35.7 GW installed RES capacity.
RES is variable, and it is difficult to predict the weather conditions, which generate new challenges in the management and operation of electrical systems, as more flexibility measures are needed to ensure safe operation and stability [1]. The energy transition from centralized power plants to distributed energy assets challenges existing utility business models due to higher market participation of customers and coordination of an increasing number of Internet of Things (IoT) devices. Simultaneously, innovations of blockchain technologies, such as the inclusions of an application layer in Ethereum have been portrayed as a potential building block to address these challenges. Despite being discussed extensively in the press, the academic community has identified substantial research gaps regarding the technology [2]. The solution in this article has a base on the development of an architecture monitoring the electricity consumption of miners using blockchain technology. The structure of the architecture is made of photovoltaic panels that are connected to the inverters and ensures the production of electricity that is monitored via the IoT Verbund device, whereas the monitoring of electricity consumption, the Fibaro smart plugs are used.

Section II of the paper presents the related work, where the primary data mining systems are described. Then section III presents the security of the equipment used in the architecture for the electrical power consumption of the building. Part IV illustrates the architecture of the system consisting of the inverters to which the photovoltaic panels are connected, together with the IoT Verbund device and the Fibaro electrical energy monitoring devices. Going forth, in section V the system architecture of the SealedGrid project is defined, and finally, in section VI, the results are concluded. 


\section{RELATED WORK}

Many advances were made in the electronic field, in the money sector regarding blockchain technology, and as well as in power management. But most important discoveries were made in the blockchain field. Mainly the most used electrical devices in the blockchain field are graphics processing units (GPUs), Field Programmable Gate Arrays FPGAs and Application-Specific Integrated Circuit (ASIC). One of the main contributors to this type of devices were Xilinx [3], and they put them on the market in 1984. One of the first FPGA had only 64 logic blocks, and each contained a two three-input Look-Up Table (LUT) and one register.

To this age, FPGAs are used for maximizing efficiency and have rigorous design requirements. They are programmed from a standpoint as software based and hardware based. These devices have power controls, like voltage scaling and Stratix adaptive body bias. One of the highest advances in FPGAs was Moore's Law into the adaptive environment, and it was suppressed, so researchers had to find a way to make it as small as possible and cheap. FPGA is a hardware circuit [3] which can be programmed to make one or $\mathrm{N}$ logical operations. FPGAs can also be considered an integrated circuit (IC). Each array on the FPGA consists of a circuit, and each group can be programmed as a logic gate or other types of parts. The interest of using an FPGA is that there is no chip and FPGA are also used for making prototypes for ASICs.

ASICs, on the other hand, are utilized in cryptocurrency systems [4], mainly in Bitcoin mining, and the SHA-256 algorithm is used for Bitcoin mining which is a hash function. SHA-256 comes from the SHA-2 family of algorithms. From this algorithm; it has been observed that it has two parts in its functionality: preprocessing and the calculated hash. Though the most widely used technology in mining are GPU's that generate the Ethereum cryptocurrency [5], a Proof-of-Work system, wherein the algorithm is called ethash. This type of algorithm uses all the available memory, and it accesses the bandwidth, so it results in a bottleneck of all computational power, and a large data structure is used in the process, under the name DAG (Directed Acyclic Graph), used while mining. Due to such circumstances, it is not possible to use and create ASICs for this type of mining, even if they have the advantage of being a general computational solution for crypto mining systems. The Smart Energy Grid (SEGs) [6] is composed of an integrated fault-tolerant ICT high technology-based system by minimizing the cost, and it focuses on data exchange between nodes. The presence of renewable energy resources, although with some limitations, allows for locally distributed energy production. The concept of MicroGrid is a particular case of SEGs. A MicroGrid system is an intelligent network that can manage renewable energy resources and energy demands from consumers. It has in its structure a house consisting of photovoltaic panels, a storage device, and user system's platform. A traditional power grid system delivers power from a few central generators to a group of users. However, smart grid systems use two-way flows of electricity and information. In this way, a smart grid system builds an energy delivery network with advantages like security, reliability, resilience, efficiency, and sustainability. In this matter, MicroGrid uses a Blockchain system. The Blockchain system is part of the ICT technology. A blockchain network provides a user-friendly application for managing power consumption. A blockchain consists of a ledger and a consensus mechanism and also it needs a cryptographic algorithm for hashing. The ledger is a register that is replicated between blocks. The consensus mechanism has the purpose of avoiding sending corrupted information further and uses proof-of-work (PoW) in which the participating nodes must solve a numerical problem [7].

The traditional transaction model (Fig.1) is centralized therefore it cannot support the increase of energy users becoming vulnerable to attacks. Thus, the Blockchain model provides a secure platform for energy management.

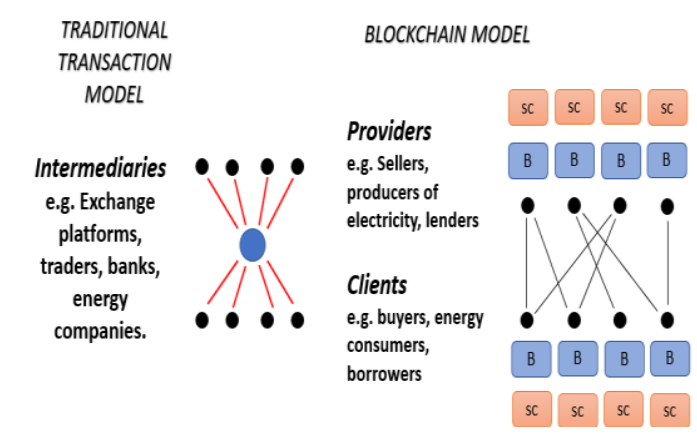

Fig 1. Transaction models

\section{SeCURity For the SEALEDGRID ARChitecture}

In this section three main subjects will be discussed, such as following:

\section{A. SealedGrid Security}

The SealedGrid architecture has a Remote Attestation Mechanism (RAM) providing identity verification, security for trust computing and allow the remote party to prove the trustworthiness of operating systems and software. Also, they enable remote authorized entities to detect changes in static files (e.g., configuration files) and runtime attestation for executable programs for improving resistance to malware. Anonymization of trusted computing operations is applicable to bidirectional communications along with the remote attestation.

In the SealedGrid build, the RAM is included in the Trusted Execution Environment (TEE), whereas the Cryptographic Storage is included in this environment. In this way, data received by the platform is protected. TEE provides confidentiality and integrity and is applied for: generation and storage of cryptographic keys, generation of random numbers, executing cryptographic operations and securing storage of the certificates.

\section{B. Z-Wave Security}

Z-Wave is a technology, widespread among Fibaro Smart Home Systems. This protocol can be used for sensor and 
actuator integration over Radio Frequency, and it can do automation of two types of services: smart home and office automation. Information about the network layer wasn't published and made available to the broad public, but the Physical and Medium Access Control layers are public. The routing protocol is based on reverse engineering, and it has been discovered that the routing and as well the topology can be changed by a third-party user. They can exploit the blind trust inherent belonging to routing nodes of that specific network.

The technology of Z-Wave enables few security characteristics which are noticeable by using a security command class, which are: source integrity, confidentiality, and data integrity service. Each application frame can be encrypted and signed, which are enclosed in a security frame. Symmetric encryption is used in this frame by utilizing the Advanced Encryption Standard, and three shared keys [8].

\section{Blockchain Technology}

The basic concept of the blockchain technology is that it uses the process of the distributed database which performs some transactions that are entirely open to the participants. The blockchain system verifies all the operations that are made, and once the transaction is done, it keeps track of the transactions, and it is not possible to destroy those records. The blockchain gives the real verification to the transactions and keeps a solid record which can never be misguided [9].

Blockchain technology can be used for all multi-level transactions where high traceability and visibility is needed. The supply chain taken as an example specifies where the lockout can influence the management and signing of contracts and enables the control of the originality of a product.

Each block contains the information about a "transaction", stored secured and encrypted. These blocks are chronologically arranged in the form of a chain with a few peculiarities [10]:

- a blockchain cannot be modified without altering all the next blocks in the chain;

- the entire chain is stored decentralized, on the computers of all participants or, finally, on many computers.

These two features of the blockchain are crucial because they make the information, which is already on hundreds or thousands or millions of computers in the world, impossible to modify. If you modify a block, all of the following blocks signal that something is wrong, and since many people already have the original version of the chain, any attempt to make a change in the chain would be immediately noticed. It is a very secure method of recording data, making sure that it cannot be modified or tampered within the future without the majority's agreement.

An example of how a blockchain splitting graph is shown in (Fig 2.), where the black blocks represent the main blockchain, the green block the original block, and the purple blocks are "orphans" that can be other chains.

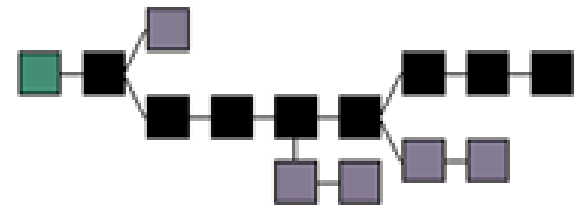

Fig. 2. Graph of blockchain splitting [11]

\section{SMart Power Management}

The purpose of experimenting with a photovoltaic system was to observe how the surplus energy made by the panels will influence the outcome of how much Ethereum will be generated by the GPU's. During the summer season of July and August 2018 the experimentations were performed and it has been observed that the photovoltaic panel system generates a significant surplus of energy. If energy produced by the panels is not enough then there will be no more Ethereum produced, meaning that the incomes brought into the company will be lower. One of the reasons for such an outcome are cloudy days with a small amount of sun energy. Also, since the opportunity to transport electricity to the national energy system did not come into effect during the experiment, the use of a mining system is the simplest and most efficient solution for using surplus energy, because it is very secure and based on a blockchain system. The energy resulting from the variation between the surplus energy and the consumed energy enables the GPU feed to generate the corresponding Ethereum.

BEIAs photovoltaic system, (Fig. 3.) consists of 66 photovoltaic panels with a maximum power of $255 \mathrm{~W}$. In order to have the best efficiency in energy transportation and transformation from Direct Current into Alternating Current the panels must be connected in series of three and then in parallel, so the installed power is approximately $17000 \mathrm{~W}$.

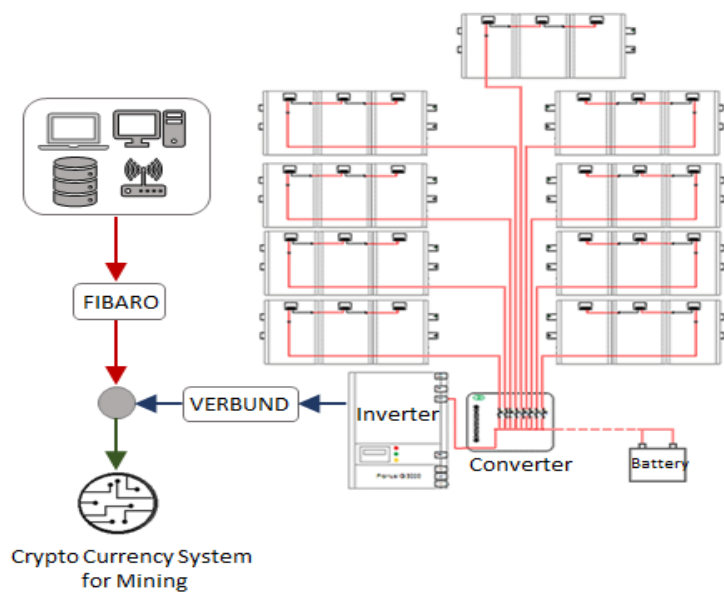

Fig. 3. BEIA RES energy power system

One of the devices used by the system is the Verbund device [12], which aims to transmit the output parameters of an inverter to a visualization platform. The Verbund equipment records the desired parameters and can forward it by dialing the address [REST/JSON] of the JSON type. This call can only be made if it is in the same wireless network. With the help of 
the IoT2020 Siemens device, the data from the Verbund equipment is being transmitted via the MQTT protocol to the mqtt.beia-telemetry.ro server with a precise sampling period. After the data was sent, the Grafana platform takes the data from the server with the link: mqtt.beia-telemetry.ro and the necessary parameters. Afterwards it displays it as a graph to make it easier to understand the events at the inverter level. The evolution in time of the electricity consumed and the produced energy monitored between 10 July and 17 August indicates the existence of surplus energy throughout the period (Fig. 4). The period is represented by 30 working days in the 9-18 hour range because it is necessary to change the number of used GPUs manually.

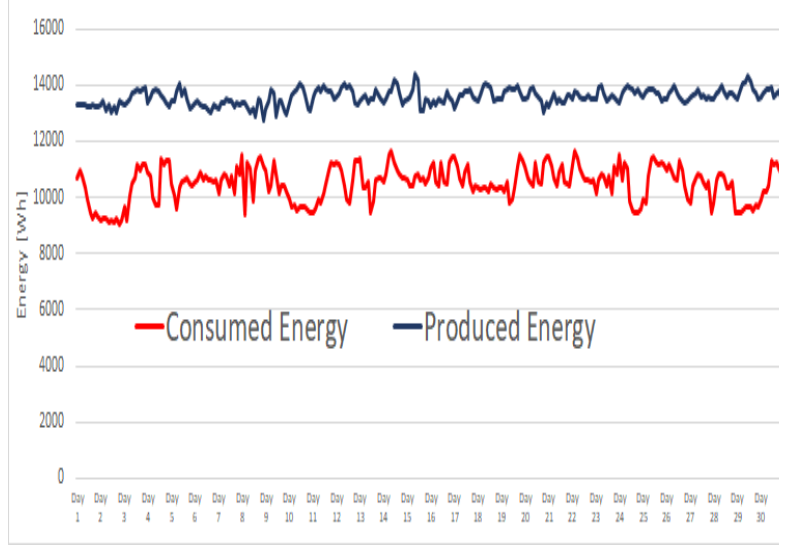

Fig. 4. The evolution in time of the electricity consumed and the produced energy

Throughout the testing phase, it has been noticed that the average energy surplus value is $3072 \mathrm{~W}$, going forth from the recorded data. The maximum amount of $4660 \mathrm{~W}$ which was monitored on day 29 and the minimum of 1660 watts on day 9 include the variation between the two values which comes from the activities performed at the headquarters, such as working days, meetings. It is noteworthy that every two weeks the consumption on the fifth working day, Friday, had an increasing tendency as seen in (Fig. 5.).

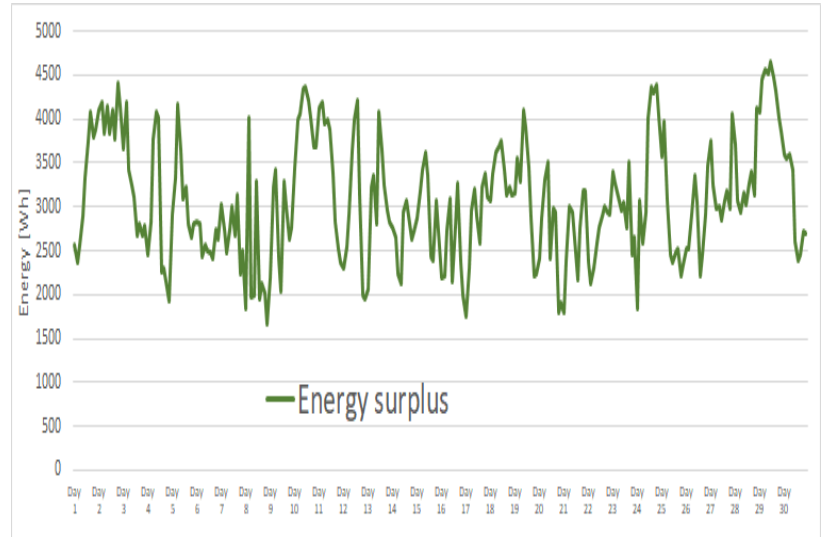

Fig. 5. The evolution in time of the energy surplus

\section{SEALEDGRID ARCHITECTURE}

The SealedGrid project [13] aims to design an architecture, as seen in (Fig. 6.), that involves equipping each participant entity with multiple modules.

The targeted clients for the SealedGrid platform are represented by the households which will use the produced energy.

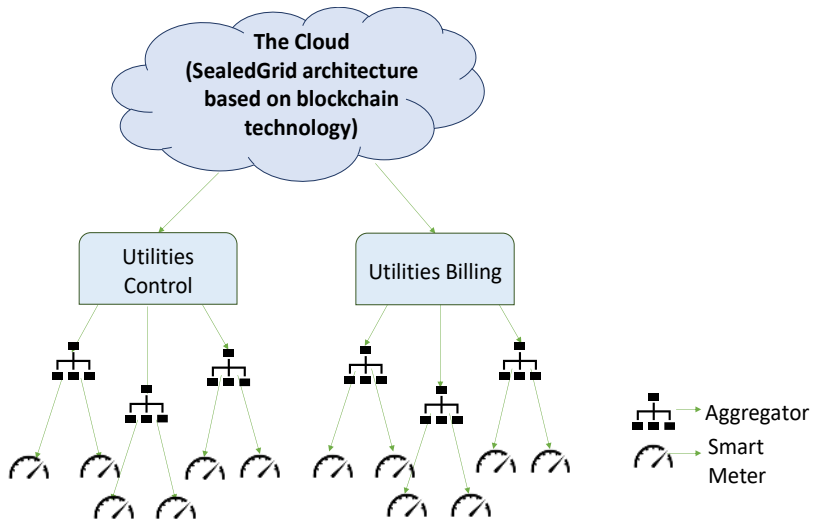

Fig. 6. SealedGrid Architecture Overview

Regarding the energy consumption, the SealedGrid project will allow the households to manage in real-time their energy consumption and even to work as energy producers.

Another objective is to implement a scalable, highly trusted and interoperable SealedGrid security platform which can be applied to existing systems, e.g., SCADA. The authentication in the platform will assume digital certificates using Web of Trust and Blockchain technologies. The SealedGrid's architecture support devices which are fully SealedGrid compliant or partial SealedGrid compliant and legacy and it has three main components, each of them having different functions:

\section{A. SealedGRID device - Smart Meter}

This device is localized in houses, or buildings and its task is to collect the readings of the electricity consumption. There can be many Smart Meters in buildings like apartment buildings having one smart meter per apartment, while detached houses have only one smart meter. The functions of this device are Applications, Federated Login, Policy Enforcement, Key Management, Authentication, TEE.

\section{B. SealedGRID device-Aggregator}

The aggregator represents the binder between the collector and the smart meters. It has the role to sum all the readings received by the meters and transmit the results to the Utility, described in the following paragraph. In this way, data becomes available without putting too much load on the Utility. The main functions of the Aggregator are Federated Login, Policy Decision, Key Management, Authentication, TEE.

\section{SealedGRID device - Utility}

The role of the Utility device is to accumulate highfrequency aggregated values, and to use them later as it is a demand for a response, or to sum these values and come after with the total grid consumption. It can also be used for billing 
by computing the total consumption of a customer at the end of a billing period. The functions of the Utility are Federated Login, Access Control/Policy Maker, Key Management, TEE.

The SealedGrid architecture [14] is especially based on the following requirements: End-to-End Security, Data-Driven Systems (Monitor $\rightarrow$ Analyse $\rightarrow$ Act), Platforms and Support Devices, Cross-Cutting Functionality, Tiered Approach, configurability, and Programmability. The Cross-Cutting functionality is necessary for the protection and the security of all layers of a smart grid. End-to-End Security provides security across all different layers and all the devices. The Tiered Approach will collect and process information both close to IoT devices and at a cloud level. Data-Driven Systems will be used for the SealedGrid project and will support the development of smart grid blockchain based monitoring platform. These kinds of systems are based on the collection and the processing of security-related data to assess risks, identify and visualize threats and produce alerts. The SealedGrid architecture should be the support for the protection of different Smart Grid platforms and devices. The last requirement, configurability, and programmability are necessary to make the architecture of SealedGrid project more flexible in accommodating different security mechanisms in a configurable and programmable fashion.

\section{CONCLUSIONS}

Conclusive in this paper the main components which were discussed were: the applicability of blockchain technology in a smart power management system, the security, and energy issues encountered in the experimentation phase and the proposed architecture in which this use-case will be used for.

As seen in the experimentation phase, the usage of surplus energy data in the mining system has resulted in the production of 0.075 Ethereum/day relative calculated on an average basis.

As future work, the system will be enabled to transmit the necessary data about the energy consumption and the energy produced in the Node-Red of the IoT2020 Siemens equipment, using a relay in order to power the GPU boards to cover up the surplus energy.

\section{ACKNOWLEDGMENT}

This work has been supported in part by UEFISCDI Romania and MCI through projects by "European Union's Horizon 2020 research and innovation program under grant agreement No. 777996 (SealedGRID project) and No. 787002 (SAFECARE project)".

\section{REFERENCES}

[1] G. Suciu, A. Pasat, R. Coanca, and S. Secu, "The adoption of photovoltaic solutions for increasing energy efficiency within SMEs," 2017 14th International Conference on Engineering of Modern Electric Systems (EMES), 2017.

[2] G. Suciu, A. Vulpe, A. Martian, S. Halunga, and D. N. Vizireanu, "Big Data Processing for Renewable Energy Telemetry Using a Decentralized Cloud M2M System," Wireless Personal Communications, vol. 87, no. 3, pp. 1113-1128, 2015.
[3] S. M. Trimberger, "Three Ages of FPGAs: A Retrospective on the First Thirty Years of FPGA Technology," Proceedings of the IEEE, vol.103, no. 3, pp. 318-331, 2015.

[4] "What Is an FPGA and Why Is It a Big Deal?" Prowess Consulting. [Online]. Available: https://www.prowesscorp.com/what-is-fpga/. [Accessed: 04-May-2019].

[5] A. R. Zamanov, V. A. Erokhin, and P. S. Fedotov, "ASIC-resistant hash functions," 2018 IEEE Conference of Russian Young Researchers in Electrical and Electronic Engineering (EIConRus), 2018.

[6] A. Pieroni, N. Scarpato, L. D. Nunzio, F. Fallucchi, and M. Raso, "Smarter City: Smart Energy Grid based on Blockchain Technology," International Journal on Advanced Science, Engineering and Information Technology, vol. 8, no. 1, p. 298, 2018.

[7] Zoican, S., Vochin, M., Zoican, R., \& Galatchi, D. Blockchain and Consensus Algorithms in Internet of Things. 2018, International Symposium on Electronics and Telecommunications ISETC 2018 (pp. 1-4). DOI: 10.1109/isetc.2018.8583923, Timisoara, Romania.

[8] J. Kaderabek, "Integration of Fibaro system to intruder and hold-up alarm systems," 2017.

[9] F. Glaser, "Pervasive Decentralisation of Digital Infrastructures: A Framework for Blockchain enabled System and Use Case Analysis," Proceedings of the 50th Hawaii International Conference on System Sciences (2017), 2017.

[10] D. Yaga, P. Mell, N. Roby, and K. Scarfone, "Blockchain technology overview," 2018.

[11] "Why Blockchain?," The Blockchain and the New Architecture of Trust, 2018.

[12] "Business Customers," VERBUND - Austria's leading electricity company. [Online]. Available: https://www.verbund.com/en-at. [Accessed: 04-May2019].

[13] SealedGRID. [Online]. Available: https://www.sgrid.eu/. [Accessed: 05-May-2019].

[14] Farnaz, "SealedGRID," SYSTEMS SECURITY LABORATORY (SSL). [Online]. Available: https://ssl.ds.unipi.gr/eventsnews/2018/12/17/sealedgrid/. [Accessed: 05-May-2019]. 